\title{
One and two dimensional interpolation schemes of atmospheric muon neutrino flux models
}

\section{Luis Salvador Miranda* and Soebur Razzaque}

Department of Physics, University of Johannesburg

P.O. Box 524, Auckland Park 2006, South Africa

E-mail: smiranda-palacios@uj.ac.za, srazzaque@uj.ac.za

\begin{abstract}
High-energy cosmic rays from all directions in space enter the Earth's atmosphere. Interaction of these cosmic rays in the atmosphere produces secondary particles, including neutrinos. Generally the behavior of atmospheric neutrino flux is accepted as a power-law function type, although the shape varies at different energies and zenith angles. Due to these complications, the fluxes from detailed numerical calculations are reported in energy and zenith angle bins. In this article we propose new interpolation schemes for the flux in energy bins or energy and zenith angle bins for any power-law type function. We apply these schemes to the atmospheric muon neutrino flux model H3a QGSJET-II-4.
\end{abstract}

5th Annual Conference on High Energy Astrophysics in Southern Africa

4-6 October, 2017

University of the Witwatersrand (Wits), South Africa

\footnotetext{
* Speaker.
} 


\section{Introduction}

Atmospheric neutrinos are secondary products of the interaction of the primary cosmic rays with the atmosphere. The primary cosmic rays, normally protons, can be very energetic, and initiate hadronic cascades with the appearance of pions and kaons. The pions and kaons further decay into muon and neutrinos (called conventional atmospheric neutrino flux). The flux of cosmic rays observed on Earth follows a power law spectrum $d N / d E \propto E^{-\gamma}$ where $\gamma=2.6$ below $30 \mathrm{GeV}$, $\gamma=2.7$ from $30 \mathrm{GeV}$ to $3 \mathrm{PeV}$ and $\gamma=3$ from $3 \mathrm{PeV}$ to EeV energy [1]. These cosmic rays interact with the atmospheric molecules and produce neutrinos with a spectrum, $d N / d E \propto E^{-p}$, where $p=\gamma-1$ [2]. However, currently more accurate methods are used to calculate the flux of neutrinos, including numerical simulations. The actual flux of neutrinos is different from the analytical approximation and it has been found that these fluxes dependent also on the cosine of the azimuthal angle [3]. Taking these effects into account one can develop accurate neutrino flux models generated using numerical simulations.

Unlike accelerator and reactor experiments, atmospheric neutrino experiments can observe the neutrino oscillation effects using fluxes from wide range of angles and energies. Monte Carlo simulations for interactions of cosmic rays in the atmosphere have been used to study the secondary particle production and the estimated atmospheric fluxes are reported in various bins of angle and energy [4]. Currently, there are many neutrino detectors (IceCube, ANTARES, Super Kamiokande, etc.) that are used for the study of physical processes involving atmospheric neutrinos. For the applications of atmospheric flux models in the study of neutrino oscillation experiments, one needs to use approximations and/or interpolation of the data from simulations. In this paper we propose an interpolation method to predict accurately the values of the atmospheric muon neutrino flux for a chosen energy value or an angle and energy values. This algorithm can be applied in general to a function with the form $\propto x^{n}$ where the index $n$ could take positive or negative values.

\section{General interpolation scheme}

The initial problem consists in that we know the value of a function at the points $x_{1}, x_{2}, \ldots, x_{n}$ but not the explicit function which allow us to calculate the value for an arbitrary point. When the chosen point is between the minimum and maximum of $x_{i}$ we have an interpolation scheme, and if the point is outside this range it is called an extrapolation scheme. The proposed functional forms to model the function include polynomials, rational functions, trigonometric functions, etc. For the polynomial method the function can be developed based on the Neville's algorithm. For a general review of the formalism and improvements, ref. [5] can be consulted. Here we only explain briefly the method for the following conditions:

a) We consider functions without strong variability so that we can interpolate only with 3 points (In many cases, like in the angle dependence of atmospheric fluxes, considering more than 3 points will not improve the calculation).

b) The fixed points $x_{1}, x_{2}$ and $x_{3}$ have the functional values $P_{1}, P_{2}$ and $P_{3}$, respectively.

According to Neville's theorem we can estimate the function using the following algorithm. For the intervals $\left[x_{1}, x_{2}\right],\left[x_{2}, x_{3}\right]$ the function can be approximated by the polynomials $P_{12}(x)$ and $P_{23}(x)$, 
respectively as:

$$
\begin{aligned}
& P_{12}(x)=\frac{\left(x-x_{2}\right) P_{1}+\left(x_{1}-x\right) P_{2}}{\left(x_{1}-x_{2}\right)} \\
& P_{23}(x)=\frac{\left(x-x_{3}\right) P_{2}+\left(x_{2}-x\right) P_{3}}{\left(x_{2}-x_{3}\right)}
\end{aligned}
$$

And the final interpolation for the complete $\left[x_{1}, x_{3}\right]$ interval takes the form:

$$
P_{123}(x)=\frac{\left(x-x_{3}\right) P_{12}+\left(x_{1}-x\right) P_{23}}{\left(x_{1}-x_{3}\right)}
$$

An improved subroutine of the method can be consulted in ref. [5].

\section{Power-law interpolation of flux in energy bins}

Cosmic rays of high energies enter the atmosphere and then interact with the environment, producing secondary particles including muons and neutrinos. The initial cosmic-ray flux extends in energy with an approximate power-law spectrum. The equations of the produced particles follow the formalism of the cascade equations. In the past these equations were solved by semi-analytical methods, but nowadays the calculation includes the use of Monte Carlo simulations and data from initial flux measurements [4]. The fluxes reported (when they depend only on energy $\phi=\phi(E)$ ) are contained in energy bins, where for each bin, we know the minimum and maximum energy values and the average flux in this energy range. For an interval with a minimum $E_{j}$ and maximum $E_{j+1}$, they are related by: $E_{j+1}=R E_{j}$, where the constant $R$ has the same value for all the samples. This flux could be fitted also with a power-law spectrum and one physical requirement is that it has to be integral preserving.

For the case of energy bin analysis we have proposed a function for the flux, the mean average value per interval and the total integral flux is not affected by the method. The interpolation algorithm consists of the following steps:

a) For a bin $j\left(\left[E_{j}, E_{j+1}\right], j=1, \ldots, n\right)$ the flux behaves like $\phi_{j}(E) \propto E^{\alpha_{j}}$, where $\alpha_{j}$ is extracted for each bin.

b) We obtain the integrated flux for the limit point of each interval as

$$
\phi_{\text {int }}\left(E_{j+1}\right)=\bar{\phi}_{j}\left(E_{j+1}-E_{j}\right)+\phi_{\text {int }}\left(E_{j}\right)
$$

where $\bar{\phi}_{j}$ is the average flux in the interval $j$. The $\phi_{\text {int }}\left(E_{1}\right)$ cannot be evaluated because we ignore the behavior of the function for lower points.

c) The integral flux is proportional to $E^{\alpha_{j}+1}$. The ratio of this quantity in the extreme points allows to obtain the desired index:

$$
\alpha_{j}=\ln \left(\frac{\phi_{\text {int }}\left(E_{j+1}\right)}{\phi_{\text {int }}\left(E_{j}\right)}\right)(\ln R)^{-1}-1
$$


d) We calculate the average energy value for the bin with the flux as weight function:

$$
\bar{E}_{j}=\left(\frac{\alpha_{j}+1}{\alpha_{j}+2}\right)\left(\frac{R^{\alpha_{j}+2}-1}{R^{\alpha_{j}+1}-1}\right) E_{j}
$$

e) With the power-law index we are preserving the integral flux. The average energy $\bar{E}_{1}$ is undefined but we can propose some initial value that is consistent with the function. Finally, to obtain the flux for a chosen energy value we perform a three point interpolation with the terms $\log (\bar{E})$ and $\log \left(\bar{\phi}_{j}\right)$ using Neville's theorem.

\section{Flux interpolation in energy and angle bins}

The flux $\phi(E, \theta)$ is considered a two-dimensional grid where each bin has the extreme points $\left[E_{j}, E_{j+1}\right]$ and $\left[\cos \theta_{k}, \cos \theta_{k+1}\right]$ with average value $\bar{\phi}_{j k}$ usually reported in the literature. We extended the procedure for the two dimensional case as

a) For the angle dependence of the flux we do not have a candidate function, rather a good approach is to define the average value:

$$
\cos \bar{\theta}_{k}=\frac{\cos \theta_{k+1}+\cos \theta_{k}}{2}
$$

b) We obtain the integral flux of each grid element using eq. (3.1) with the replacement $\bar{\phi}_{j} \longrightarrow \bar{\phi}_{j k}$.

c) For the $\cos \theta$ value chosen to interpolate the logarithm of the integral and differential fluxes with the method of the section (2) and reference points of eq. (4.1), we obtain a group of fluxes with only energy dependence.

d) Follow the last section after step c) with the new energy dependence fluxes to obtain the final interpolation for the energy value chosen.

\section{Results}

The atmospheric muon neutrino flux is measured at the detectors, after crossing the Earth, to study physical properties of neutrinos. The survival and oscillation probabilities after crossing the Earth affect the magnitude of the flux intensity. The phenomena require study of the initial atmospheric flux. The main predictions in recent studies came from the numerical HKKM model with the H3a knee correction for primary models $[6,3,7]$, where two different hadronic models are considered, namely QGSJET-II-4 [8] and SIBYLL2.3 [9]. The two different hadronic models provide the minimum and maximum values for the flux considered. These and other models are revised and discussed in the literature [10]. The flux data from simulations are provided in bins of energy and cosine of zenith angle. For the energy, data are given from $10^{2}-10^{6} \mathrm{GeV}$ with the factor $R=10^{4 / 149}$. For the angular bins, the cosine of the zenith angle has a maximum value of 0.2 and minimum of -1 with $\Delta \cos \theta=1.2 / 39$. The flux data are provided for the neutrino and anti-neutrino channels separately and we have added these two contributions to consider the total initial flux. In this work, we have applied our interpolation method to the H3a QGSJET-II-4 model. 


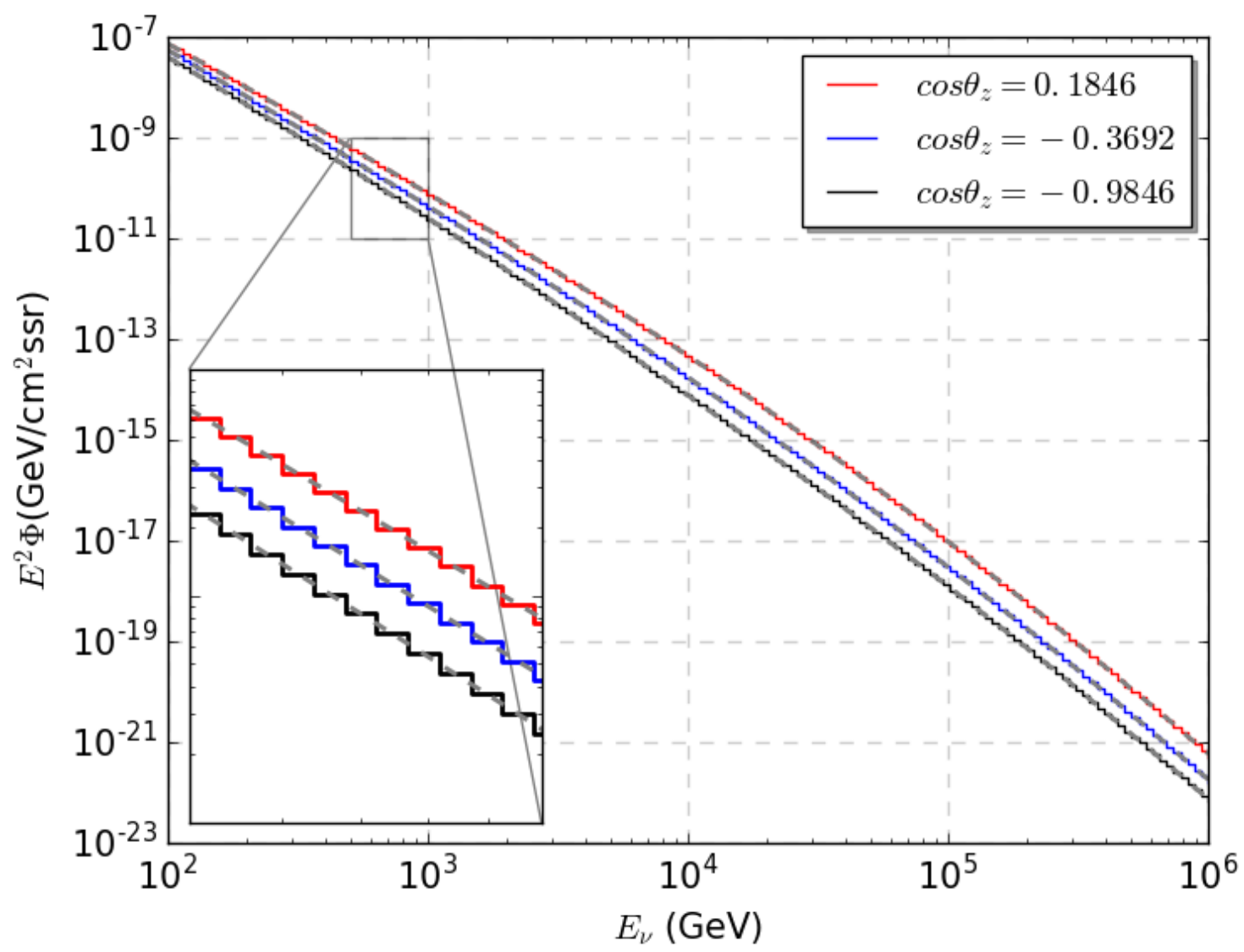

Figure 1: Interpolation Model (dashed gray lines) applied to three different angle bins for the H3a QGSJETII-4 atmospheric neutrino model data (red, blue and black histograms) in $10^{2}-10^{6} \mathrm{GeV}$ energy range.

In Figure 1 we have compared our interpolation to simulated data in 3 different zenith angle bins and over $10^{2}-10^{6} \mathrm{GeV}$ energy range. The zenith angle bins are cosine angle [0.169231,0.2] with mean value $\cos \bar{\theta}=0.1846,[-0.384615,-0.353846]$ with mean value $\cos \bar{\theta}=-0.3692$ and $[-1,-0.9692]$ with mean value $\cos \bar{\theta}=-0.9846$. Our predictions due to interpolation (dashed gray lines) show a good fit to the data set and a form of power law type that is common for atmospheric fluxes. Thus our algorithm can be useful for extracting the information given by the simulation models and provide the value of the fluxes for any given energy and angle.

\section{Summary}

In this paper we have proposed an interpolation model for the functions of power-law type. In turn, the method was applied to one of the recent atmospheric neutrino flux models. We have shown that the interpolation method adjusts quite well and we can finally predict the flux value for any energy and angle within the established ranges. We have also proposed a model that can be applied to any function of power-law type, which can be of increasing or decreasing form. Also, it is possible to extend the model for the azimuth angle-dependence of atmospheric neutrino flux, in 
which case a 3-variable interpolation problem is addressed. This tool will be useful in later studies that we do with sterile neutrino models.

\section{References}

[1] K. Kotera, A.V. Olinto, The Astrophysics of Ultrahigh-Energy Cosmic Rays, Phys. Rev. D 49, 119 (2011).

[2] Thomas K. Gaisser Cosmic Rays and Particle Physics, Cambridge University Press, Cambridge 1990.

[3] M. Honda et al., Calculation of atmospheric neutrino flux using the interaction model calibrated with atmospheric muon data, Phys. Rev. D 75043006 (2007) [arXiv: astroph/ 0611418 ].

[4] T. K. Gaisser and M. Honda, Flux of atmospheric neutrinos, Ann. Rev. Nucl. Part. Sci. 52, 153 (2002).

[5] Press W. H. et al., Numerical Recipes in Fortran 77, EXXON Research and Engineering Company, New York 1992.

[6] T. Sanuki et al., Study of cosmic ray interaction model based on atmospheric muons for the neutrino flux calculation, Phys. Rev. D 75043005 (2007) [arXiv: astroph/ 0611201 ].

[7] T. K. Gaisser et al., Cosmic Ray Energy Spectrum from Measurements of Air Showers, Front.Phys.China 8748 (2013) [arXiv: 1303.3565 ].

[8] S. Ostapchenko, Monte Carlo treatment of hadronic interactions in enhanced Pomeron scheme: I. QGSJET-II model, Phys. Rev. D 83014018 (2011) [arXiv: 1010.1869 ].

[9] F. Riehn et al., A new version of the event generator Sibyll PoS ICRC2015 558(2016) [arXiv:1510.00568].

[10] https://dspace.mit.edu/handle/1721.1/98078. 